Louisiana State University

LSU Digital Commons

$11-26-2019$

\title{
Amphiphilic Polypeptoids Rupture Vesicle Bilayers to Form Peptoid-Lipid Fragments Effective in Enhancing Hydrophobic Drug Delivery
}

\author{
Yueheng Zhang \\ Tulane University \\ Zahra Heidari \\ Tulane University \\ Yang Su \\ Tulane University \\ Tianyi Yu \\ Louisiana State University \\ Sunting Xuan \\ Louisiana State University
}

See next page for additional authors

Follow this and additional works at: https://digitalcommons.Isu.edu/chemistry_pubs

\section{Recommended Citation}

Zhang, Y., Heidari, Z., Su, Y., Yu, T., Xuan, S., Omarova, M., Aydin, Y., Dash, S., Zhang, D., \& John, V. (2019). Amphiphilic Polypeptoids Rupture Vesicle Bilayers to Form Peptoid-Lipid Fragments Effective in Enhancing Hydrophobic Drug Delivery. Langmuir, 35 (47), 15335-15343. https://doi.org/10.1021/ acs.langmuir.9b03322

This Article is brought to you for free and open access by the Department of Chemistry at LSU Digital Commons. It has been accepted for inclusion in Faculty Publications by an authorized administrator of LSU Digital Commons. For more information, please contact ir@lsu.edu. 


\section{Authors}

Yueheng Zhang, Zahra Heidari, Yang Su, Tianyi Yu, Sunting Xuan, Marzhana Omarova, Yucel Aydin, Srikanta Dash, Donghui Zhang, and Vijay John 


\section{Amphiphilic Polypeptoids Rupture Vesicle Bilayers to Form Peptoid-Lipid Fragments Effective in Enhancing Hydrophobic Drug Delivery}

Yueheng Zhang ${ }^{1}$, Zahra Heidari ${ }^{1}$, Yang $\mathrm{Su}^{1}$, Tianyi $\mathrm{Yu}^{2}$, Sunting Xuan ${ }^{2}$, Marzhana Omarova ${ }^{1}$, Yucel Aydin ${ }^{3}$, Srikanta Dash ${ }^{3}$, Donghui Zhang ${ }^{2 *}$, Vijay John ${ }^{1 *}$

1. Department of Chemical and Biomolecular Engineering Tulane University, 6823 St. Charles Ave, New Orleans, LA 70118, USA

2. Department of Chemistry

Louisiana State University, Baton Rouge, Louisiana 70803, USA

3. Department of Pathology and Laboratory Medicine

Tulane University, 1430 Tulane Ave, New Orleans, Louisiana 70112, USA

*Corresponding authors.

Vijay John, Phone: (504)865-5883 Fax: (504) 865-6744 Email: vj@ tulane.edu.

Donghui Zhang, Phone: (225)578-4893 Email: dhzhang@1su.edu 


\section{ABSTRACT}

Peptoids are highly biocompatible pseudo-peptidic polyglycines with designable substituents on the nitrogen atoms. The therapeutic and drug-carrying potential of these materials requires a fundamental understanding of their interactions with lipid bilayers. In this work, we use amphiphilic polypeptoids with up to 100 monomeric units where a significant fraction (26\%) of the nitrogen atoms are functionalized with decyl groups (hydrophobes) that insert into the lipid bilayer through the hydrophobic effect. These hydrophobically modified polypeptoids (HMP) insert their hydrophobes into lipid bilayers creating instabilities that lead to the rupture of vesicles. At low HMP concentrations, such rupture leads to the creation of large fragments which remarkably anchor to intact vesicles through the hydrophobic effect. At high HMP concentrations all vesicles rupture to smaller HMP-lipid fragments of the order of $10 \mathrm{~nm}$. We show that the technique for such nanoscale polymer-lipid fragments can be exploited to sustain highly hydrophobic drug species in solution. Using the kinase inhibitor, Sorafenib as a model drug, it is shown that HMP-lipid fragments containing the drug can efficiently enter a hepatocellular carcinoma cell line (Huh 7.5) indicating the use of such fragments as drug delivery nanocarriers. 


\section{INTRODUCTION}

Liposomes are a class of lipid vesicles that have shown significant promise as drug delivery systems since the lipid bilayers mimic cell membranes leading to endocytosis by cells. Thus, these systems can be designed to deliver hydrophilic molecules through encapsulation in the water core of the liposome and hydrophobic drug species through integration into the lipid bilayer. ${ }^{1-3}$ The delivery of hydrophobic drug species is of specific relevance to cancer therapy ${ }^{4-5}$ and incorporation into the hydrophobic environment of lipid nanostructures such as liposomes, bicelles, solid lipid nanoparticles represent new routes to delivery. ${ }^{6-10}$

The interaction of vesicle lipid bilayers with a class of amphiphilic biomimetic polymers known as polypeptoids is the subject of this study. Peptoids are a class of pseudo-peptidic polyglycines with substituents on the nitrogen atoms. ${ }^{11-12}$ These polymers are of much interest due to their highly designable structure and their structural similarity to peptides. ${ }^{13-14}$ Without extensive hydrogen bonding and stereogenic centers along the backbone due to the $\mathrm{N}$-substitution, peptoids have a more flexible conformation and therefore enhanced resistance against proteolysis. ${ }^{15-17}$ In addition, peptoids are biocompatible, and are therefore potentially useful in biomedical applications such as drug delivery devices, tissue engineering materials, and smart coatings. ${ }^{18-20}$

Our focus is the use of hydrophobically modified polypeptoids (HMPs) with up to 100 monomer units, and their interaction with lipid bilayers. The hydrophobic modification is the functionalization of the nitrogen with alkyl groups which constitute the hydrophobes on an otherwise water-soluble backbone. Thus, we specifically use the random copolymer shown in Figure 1a, containing $74 \%$ of the neutral $N$-methoxyethyl groups (MeOEt) and $26 \%$ of $N$-n-decyl (C10) groups along the backbone (Supporting Information S1, Scheme S1). The molecular weight 
a

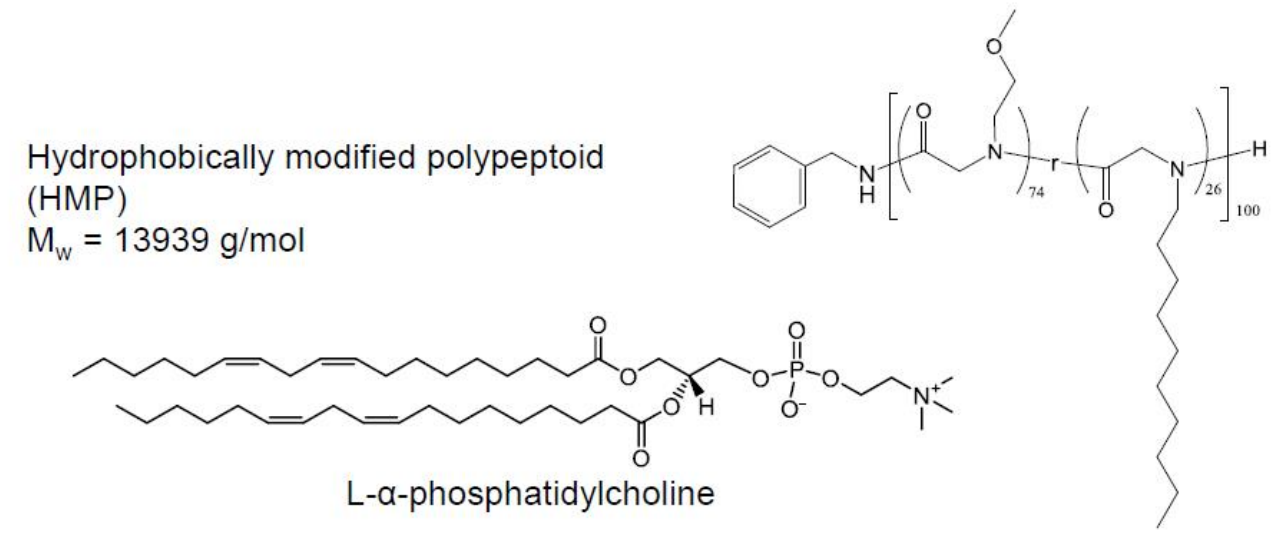

b

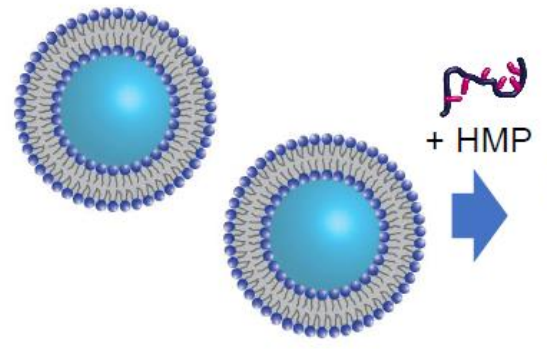

Liposomes

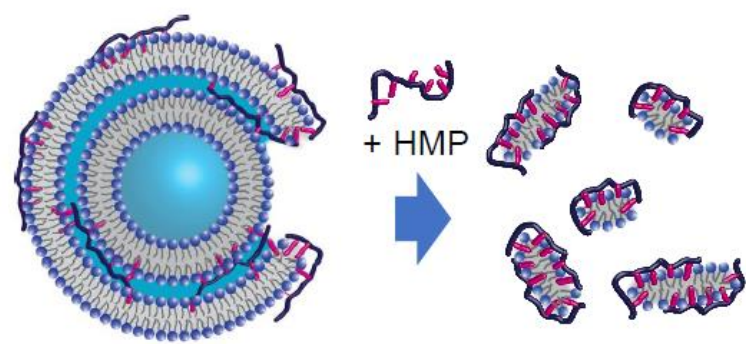

HMP-lipid fragments

Figure 1. (a) Structure of the hydrophobically modified polypeptoid (HMP) and the phospholipid (L- $\alpha$-phosphatidylcholine or PC) used to form vesicles. (b) Schematic showing disruption of liposomes. At low HMP concentrations some of the liposomes are ruptured to large fragments which attach to intact liposomes. At high HMP concentrations all liposomes are ruptured to HMP-lipid fragments.

of the polymer is $13.9 \mathrm{kDa}$ (Supporting Information S2, Figure S1) with a polydispersity index (PDI) of 1.08 (Supporting Information S3, Figure S2). The polymer remains water soluble at this level of hydrophobic substitution. The reason to use HMPs is based on the well-known phenomenon of hydrophobe insertion into lipid bilayers through the hydrophobic effect. ${ }^{21-22}$ In recent work from our laboratories we showed that HMPs significantly modify liposome characteristics through such hydrophobe insertion. ${ }^{23}$ Figure $1 \mathrm{~b}$ is a synopsis of our earlier work ${ }^{23}$ wherein we found that at low HMP concentrations some of the liposomes are ruptured by HMPs into large fragments or HMP-lipid fragments. Interestingly, these fragments anchor to intact liposomes to form additional layers on the remaining intact liposomes through a bridging effect 
where some of the hydrophobes are in one lipid layer while others are in the adjoining layer. HMP thus serves as a connecting glue to bridge lipid bilayers. At higher HMP concentrations, all liposomes are ruptured, resulting in the formation of smaller HMP-lipid fragments. ${ }^{23}$

These observations are useful as they imply the ability to create small lipid-based structures for drug delivery and the potential to attach such fragments to cell membranes thus facilitating entry into the cell. Accordingly, the current study is the next level of understanding focusing on the formation of HMP-lipid fragments and their potential to encapsulate hydrophobic drugs for cell entry. Figure 2 schematizes the objectives of the current study to generate and characterize HMP-lipid fragments. Path I is the generation of such fragments through rupturing of liposomes preformed through well-established procedures of thin film plating of lipids, hydration of these thin films to form large vesicles which are extruded to liposomes. While the formation of HMPlipid fragments by adding HMP to preformed liposomes has been established in our recent work, ${ }^{23}$

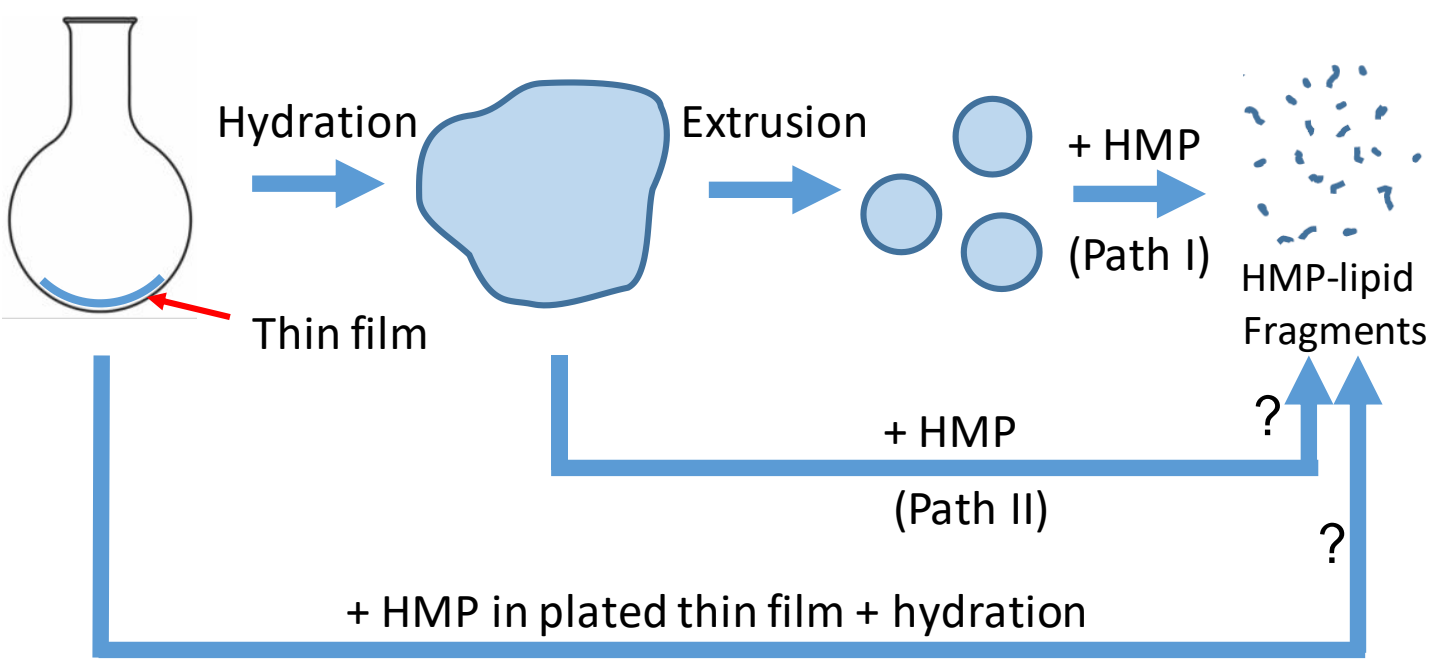

(Path III)

Figure 2. Schematic of generating HMP-lipid fragments through different paths. Path I illustrates the generation of HMP-lipid fragments by interrupting lipid bilayers of extruded liposomes. Path II shows the hypothesis to generate HMP-lipid fragments by interrupting lipid bilayers of giant vesicles. Path III describes the hypothesis to form HMP-lipid fragments by hydrating a thin film consisting of HMPs and lipids. 
we seek to establish the shorter routes to raft formation by deleting the extrusion step and directly contacting the unextruded vesicles as shown in Path II. Path III describes an even more facile approach where HMP is integrated into the plated thin films of lipids. The question then arises as to whether hydration of the thin film will directly lead to raft formation. Finally, we characterize the ability of these fragments to sustain a hydrophobic drug, Sorafenib, and show the potential of these fragments to enter cells of a hepatocellular carcinoma cell line (Huh 7.5). 


\section{EXPERIMENTAL SECTION}

\section{Materials}

L- $\alpha$-phosphatidylcholine (PC, >95\%, from soy) and 1,2-dioleoyl-sn-glycero-3phosphoethanolamine- $N$-(carboxyfluorescein) (PECF, ammonium salt, 99\%) were purchased from Avanti Polar Lipids. Deuterium oxide (>99.9\%) was purchased from Cambridge Isotope Laboratories. Dimethyl sulfoxide (99.5\%) was purchased from Sigma-Aldrich. Sorafenib $(>99 \%)$ was purchased from Eton Bioscience (San Diego, CA). All chemicals were used as received unless otherwise noted. $N$-methoxyethyl- $N$-carboxyanhydride ( $N$-methoxyethyl NCA) and $N$-decyl- $N$-carboxyanhydride ( $N$-decyl NCA) were synthesized through previously reported

procedures as shown in Supporting Information Scheme S1. ${ }^{23-25}$ Hoechst dye (H33342) was purchased from Thermo Scientific, Germany. Huh-7.5 cells were obtained from the laboratory of Dr. Srikanta Dash (Department of Pathology and Laboratory Medicine, Tulane University Health Sciences Center, Louisiana) and the cells were cultured in Dulbecco's Modified Eagle Medium (DMEM; Invitrogen, San Diego, CA) with high glucose supplemented with nonessential amino acids, sodium pyruvate, and 5\% fetal bovine serum.

\section{Methods}

Synthesis and Characterization of Polypeptoids

The HMPs were synthesized through benzyl amine-initiated ring-opening polymerization of the corresponding R-NCA monomers as reported previously. ${ }^{23}$ Copolymerization of $\mathrm{N}$ methoxyehtyl NCA and $N$-decyl NCA yields a random copolymer of $N$-methoxyethyl glycine units and $N$-decyl glycine units. The composition of the HMP backbone can be adjusted by controlling the ratio of the two R-NCA monomers. In a typical synthesis, stock solutions of $N$-methoxyethyl 
NCA (1.3 mL, 0.4 M, $0.52 \mathrm{mmol})$ and $N$-decyl NCA (0.3 mL, $0.4 \mathrm{M}, 0.13 \mathrm{mmol})$ in THF were mixed together with a benzyl- $\mathrm{NH}_{2} / \mathrm{THF}$ stock solution $(70 \mu \mathrm{L}, 92.7 \mathrm{mM}, 6.5 \mu \mathrm{mol})$. The mixture was heated to $50{ }^{\circ} \mathrm{C}$ for 48 hours. ${ }^{1} \mathrm{H}$ NMR spectroscopy was used to check the conversion. The polymer was precipitated out by excess hexanes, collected by filtration, and dried under vacuum to obtain the final polypeptoid as a white solid (61.6 $\mathrm{mg}, 88.2 \%$ yield).

${ }^{1}$ H NMR spectra of HMP (Supporting Information S2, Figure S1) were obtained using a Bruker AV-400 Nanobay spectrometer at $298 \mathrm{~K}(400 \mathrm{MHz})$. Chemical shifts $(\delta)$ were referenced to proton impurities in $\mathrm{CD}_{2} \mathrm{Cl}_{2}$. The exact composition of HMP was therefore determined. The integration of the methylene groups in the backbone relative to the integration of benzyl end-group yields a degree of polymerization (DP) of 100, while integration of methyl groups of $N$-decyl side chains relative to the integration of benzyl end-group confirms a fraction of $26 \mathrm{~mol} \% \mathrm{~N}$-decyl glycine units in the polymer backbone. The sample for size exclusion chromatography (SEC) analysis was prepared by diluting $130 \mu \mathrm{L}$ reaction mixture of HMP in $1 \mathrm{~mL}$ DMF containing 0.1 M LiBr. The analysis was carried out on an Agilent 1200 system equipped with three Phenomenex $5 \mu \mathrm{m}, 300 \times 7.8 \mathrm{~mm}^{2}$ columns [100 ̊, $1000 \AA$ and Linear (2)], a differential refractive index (DRI) detector (Wyatt OptilabrEX) with a $690 \mathrm{~nm}$ light source, and a multi-angle light scattering detector (Wyatt DAWN EOS, GaAs $30 \mathrm{~mW}$ laser at $\lambda=690 \mathrm{~nm}$ ). $0.1 \mathrm{M} \mathrm{LiBr}$ in DMF solution was used as the eluent. The flow rate was set at $0.5 \mathrm{~mL} \mathrm{~min}^{-1}$. The column and detector were operated at 22 ${ }^{\circ} \mathrm{C}$. The polydispersity index (PDI) was obtained with a calibration curve constructed by polystyrene standards. A monomodal peak and a PDI of 1.08 indicate a narrow distribution of polymer molecular weight (Supporting Information S3, Figure S2).

\section{Preparation of Liposomes}


The liposomes were prepared by the thin-film hydration technique where the lipids are first dissolved in an organic solvent and then evaporated to form a lipid thin film. ${ }^{26-27}$ Typically, 100 mg PC lipid was dissolved in $15 \mathrm{~mL}$ chloroform and methanol mixture $(2 / 1, \mathrm{v} / \mathrm{v})$ in a round bottom flask. The solvent was then evaporated on a rotavapor (Buchi R-205) at room temperature at 100 mbar for 3 hours to form a thin lipid film. The film was further treated in vacuum at 6 mbar for 30 min to remove residual solvent. The formed thin lipid film was then hydrated using DI water at 50 ${ }^{\circ} \mathrm{C}$ which yielded a suspension of large lipid vesicles. Fluorescent large vesicles were prepared by adding $0.5 \mathrm{mg}$ fluorescein-tagged lipid (PECF) to the organic solution of lipids followed by the same manner. The vesicle suspension was extruded 21 times through polycarbonate membrane with a pore size of $100 \mathrm{~nm}$ to downsize the unextruded vesicles into small unilamellar vesicles with an average diameter of $100 \mathrm{~nm}$. In all further discussion, the term liposome will apply to the small extruded lipid vesicles. We recognize that the term liposome is general referring to lipid vesicles, but for clarity we use the term unextruded vesicle to represent the large lipid vesicles formed after hydration, and the term liposome to represent smaller lipid vesicles obtained after extrusion. The liposome suspension was then diluted to $0.5 \mathrm{wt} \%$ (LIPO). To prepare drug containing liposomes, Sorafenib was added to the lipid formulation and was dissolved together with the lipids in the organic solvent followed by the same procedure. ${ }^{27}$ All concentrations are reported in wt\% unless otherwise mentioned.

We also prepared liposomes using a freeze-thaw technique to attempt to improve the unilamellarity and size distribution. In the freeze-thaw technique. First, large vesicles were prepared via thin film hydration method. After the hydration step the unextruded vesicles were subjected to 10 cycles of freeze-thaw procedure to increase the volume of trapped aqueous phase and thus reduce the lamellarity of the vesicles. ${ }^{28-29}$ Thus, the lipid suspension was transferred into 
polypropylene conical centrifuge tubes and was first submerged into a liquid nitrogen bath for 5 $\min$. The suspension was then immediately plunged into water bath at $50^{\circ} \mathrm{C}$ for $5 \mathrm{~min}$. The $10 \mathrm{~min}$ period of submersion into liquid nitrogen and water was considered as one cycle and the procedure was repeated for a total of 10 cycles. After the freeze-thaw the vesicle suspension was extruded through $100 \mathrm{~nm}$ polycarbonate membrane to form liposomes.

\section{Rupture of Liposomes using HMPs}

The samples were prepared by mixing liposome stock solution (0.5\%) with polypeptoid stock solutions ( $0.5 \%$ and $1 \%$ in DI water) at 1:1 volume ratio. Samples were prepared from either large unextruded vesicles (LVES) or extruded vesicles (liposomes -LIPO). The samples are therefore denoted as LVES-HMP1, LVES-HMP2, LIPO-HMP1, and LIPO-HMP2, respectively, where the number indicates the mass ratio of HMP to lipid. Liposomes incubated with DI water was also prepared as the blank control, denoted as LIPO. Samples were incubated for at least 12 hours at room temperature prior to instrumental characterization. The HMP-LIP fragments (or fluorescence-tagged fragments HMP-PECF) were also obtained by hydrating the thin film of HMPs and lipids which were plated out together from the chloroform/methanol solution of HMPs and lipids. To prepare drug loaded HMP-LIP fragment (HMP-LIP-SF), SF was added to the

chloroform/methanol solution of HMPs and lipids. $\mathrm{D}_{2} \mathrm{O}$ was used instead of $\mathrm{H}_{2} \mathrm{O}$ for ${ }^{31} \mathrm{P}$ NMR and ${ }^{1}$ H NMR spectra (Bruker AVANCE $300298 \mathrm{~K}, 120 \mathrm{MHz}$ ).

\section{Formation of HMP-lipid fragments by thin film hydration}

To prepare HMP-lipid fragments through the thin film hydration process, HMP and lipid were dissolved together in a chloroform/methanol $(2 / 1, \mathrm{v} / \mathrm{v})$ mixture. The weight ratio of HMP to lipid was controlled at 2:1. The solvent evaporation step followed the same procedure and 
conditions as the general procedure to prepare lipid vesicles, resulting in a thin film consisting of HMP and lipid at 2:1 ratio rather than the usual exclusively lipid thin film used to form vesicles after hydration. The HMP-lipid thin film was then hydrated using DI water at room temperature, to yield a clear solution. A suspension of Sorafenib (SF) loaded HMP-lipid fragments was also made by adding SF to the chloroform/methanol solution of HMPs and lipids, followed by the same evaporation and hydration steps as empty HMP-lipid fragments. In this study, the weight ratio of lipid to SF was controlled at 1:0.024. We call this HMP-Lip-SF-P to recognize that the fragment contains HMP, lipids and sorafenib that were directly formed by hydrating a plated out film of these species.

\section{Electron and Optical Microscopy Characterization}

CryoTEM imaging was carried out on an FEI G2 F30 Tecnai TEM operating at $150 \mathrm{kV}$. The sample for CryoTEM imaging was prepared on a lacey carbon coated copper grid (200-mesh, Electron Microscopy Sciences) using an automated plunging station (FEI Vitrobot). $12 \mu \mathrm{L}$ of the sample solution was applied to the grid. The excess liquid was blotted by attached blotting papers

for 2 seconds to form a thin sample film which was immediately vitrified by being plunged into liquid ethane. The grid with sample cryogenically immobilized was transferred onto a single tilt cryo specimen holder for imaging. The optical microscopy images were taken with a Nikon A1 confocal microscope. $20 \mu \mathrm{L}$ sample was pipetted onto a standard microscope slide. $488 \mathrm{~nm}$ laser was used to excite the fluorescence-tagged samples.

\section{Dynamic Light Scattering (DLS)}

Dynamic light scattering (DLS) was used to characterize the size of the assemblies before and after the addition of HMPs. Each DLS sample was diluted with $\mathrm{NaCl}$ solution to form a 
solution with $0.15 \%$ lipid and $50 \mathrm{mM} \mathrm{NaCl}$, regardless HMP concentration. The solution was then filtered through a PTFE filter $(0.22 \mu \mathrm{m}$, Whatman) 3 times except for the unextruded vesicle sample. The DLS particle sizing measurements were carried out on a Brookhaven Instrument (90Plus PALS) which is equipped with a $640 \mathrm{~nm}$ laser. For each sample, 10 measurements of 100 s were performed at $25^{\circ} \mathrm{C}$, and the scattering signal was collected at $90^{\circ}$. The data were analyzed using CONTIN method embedded in BIC Particle Solutions (v3.1) software. The lognormal median diameter was reported as effective diameter or hydrodynamic diameter $\left(\mathrm{D}_{\mathrm{H}}\right)$.

\section{Contact of HMP-Lipid Fragments with Living Cells for uptake}

1, 2-dioleoyl-sn-glycero-3-phosphoethanolamine-N-carboxyfluorescein (PECF) was used as the fluorescent probe to observe the fusion of the HMP-lipid fragment to Huh 7.5 (human hepatocyte) cell line. The Huh 7.5 cells were cultured in Dulbecco's modified Eagle's medium supplemented with $10 \%$ fetal bovine serum and $1 \%$ penicillin/streptomycin and incubated at $37^{\circ} \mathrm{C}$ and $5 \% \mathrm{CO}_{2}$. Cells were seeded in 6 -well plates at a density of $1 \times 10^{5}$ cells per well. $5 \mu \mathrm{L}$ of the PECF-labeled HMP-lipid was added to the growth media. After 4 hours of incubation, the cells were washed with PBS 3 times followed by cell fixation using $4 \%$ paraformaldehyde in PBS. Unbound and extracellular PECF-labeled HMP-lipid was rinsed off with sterilized PBS solution. Following this step, Hoechst 33342 (Invitrogen) in PBS $\left(2 \mu \mathrm{g} \mathrm{mL}^{-1}\right)$ was used to stain the nucleus

of the cells for visualization. ${ }^{30}$ Fluorescence images of cells were captured using an Olympus IX70 microscope and an Olympus DP-71 digital camera. The negative control sample was prepared by dissolving $50 \mathrm{mM}$ PECF in DMSO and then diluted with the PBS buffer. 


\section{RESULTS AND DISCUSSION}

Vesicle Rupture Induced by HMPs

The background for our current work lies in Figure 3 (a-c) which briefly summarizes our findings on the interaction of HMP with fully extruded $100 \mathrm{~nm}$ liposomes. ${ }^{23}$ Thus, Figure $3 \mathrm{a}$ shows liposomes in the range of $100 \mathrm{~nm}$ that are made through the traditional methods of lipid film formation, hydration to large lipid vesicles and subsequent extrusion to form relatively uniform sized smaller vesicles which we term as liposomes. Upon adding HMPs to a level of 0.25 $\mathrm{wt} \%$, thes liposomes remarkably transform to layered structures (Figure 3b) where some of the layers are incomplete (yellow arrows of Figure 3b). Through a combination of small-angle neutron scattering and CryoTEM in our recent work, the observations can be understood. ${ }^{23}$ First, the insertion of a high local concentration of hydrophobes into liposome bilayers leads to the disruption of some of the liposomes into large fragments. Since hydrophobe insertion into lipid bilayers is a dynamic phenomenon, some of the hydrophobes on the polymer-lipid fragments come free and reinsert into the bilayer of intact liposomes as shown schematically in Figure 1b. This leads to the formation of the layered structures on liposomes, but the number density of liposomes in solution is reduced (as was shown by the loss of intensity in the low q region of the SANS scattering profile. ${ }^{23}$ With an increase in the concentration of the HMP, all liposomes become disrupted into fragments. Thus, Figure $3 c$ is a CryoTEM image of $0.25 \%$ extruded liposomes incubated in 0.5\% HMP, where all liposomes have been disrupted into small fragments in the 10 $\mathrm{nm}$ size range. The DLS data shown in Figure $3 \mathrm{~d}$ additionally verifies this rupture with liposomes of mean hydrodynamic diameter $126 \mathrm{~nm}$ breaking into fragments with mean hydrodynamic diameter $10.8 \mathrm{~nm}$ and polydispersity index 0.16 , indicating a moderately polydispersed size distribution of the HMP-lipid fragments. Since these fragments represent broken-off lipid layers 
which are sustained in solution with HMP, we refer to them as HMP-lipid fragments. We note that the edges of these fragments energetically cannot have exposed lipid tails and therefore expect that the HMP polar groups cover these edges or that lipids rearrange at the edges to present a hydrophilic exterior.
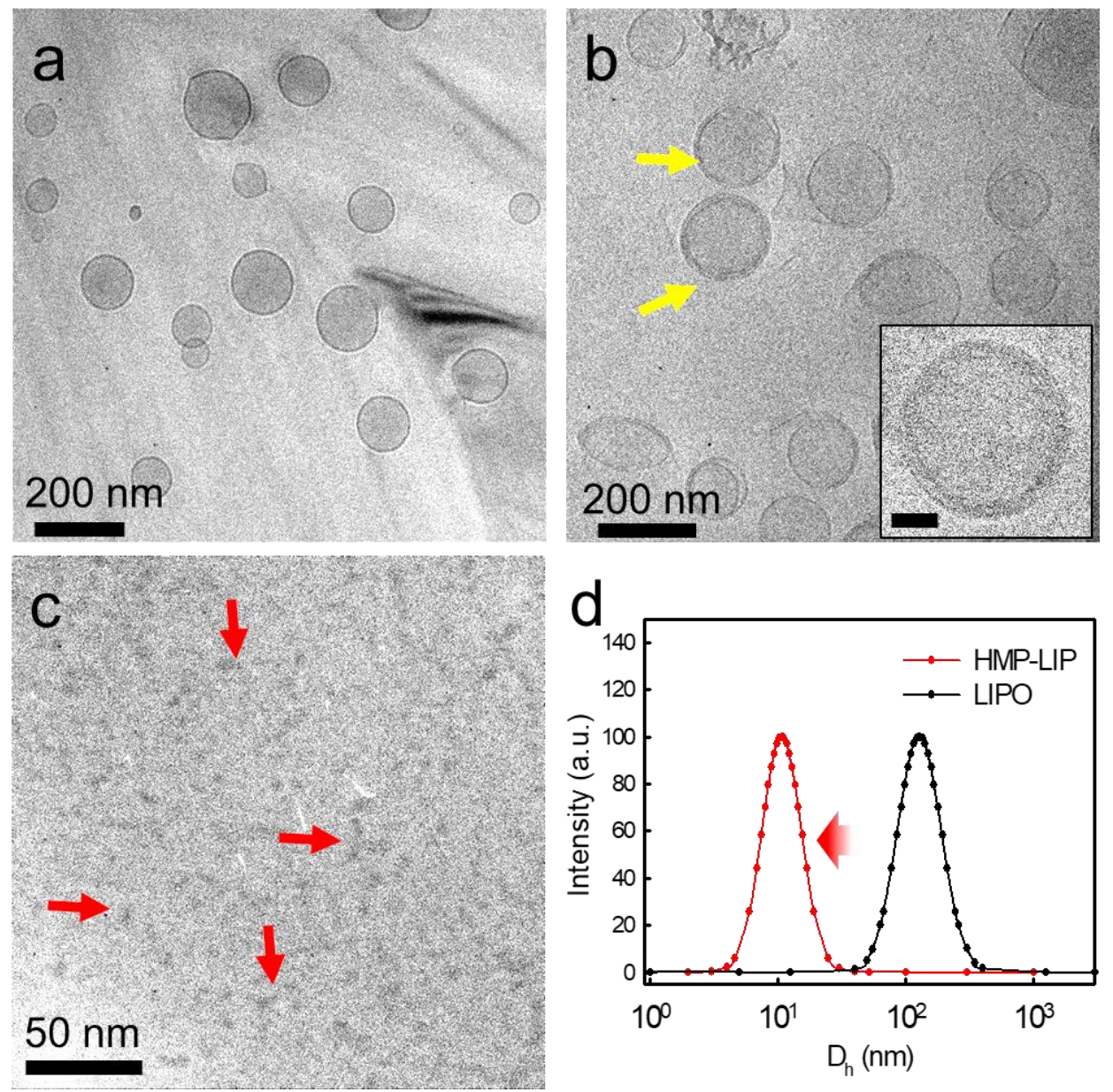

Figure 3. CryoTEM images of $0.25 \%$ extruded liposomes incubated with HMPs at different concentrations and the size distributions of liposome and HMP-lipid fragment. (a) Unilamellar liposomes with the size of $100 \mathrm{~nm}$ are formed without the addition of HMP (LIPO). (b) Liposomes with multiple layers are formed with the addition of $0.25 \%$ HMP. Arrows point out liposomes with multilayered structures. Scale bar in the inset is $50 \mathrm{~nm}$. (c) Liposomes are broken into small fragments with the addition of 0.5\% HMP (HMP-LIP). (d) The size distribution shows an effective liposome diameter of $126.4 \mathrm{~nm}$, which shifts to $10.8 \mathrm{~nm}$ with polydispersity index 0.16 after the addition of $0.5 \%$ HMP leading to the formation of HMP-LIP fragment. 
The question that immediately arises is whether such HMP-lipid fragments can be obtained without the extrusion step, thus starting from large vesicular structures as described by Path II in Figure 2. Figure 4a is the CryoTEM image of micron-sized large vesicles formed directly from the hydration step without any extrusion. After the addition of HMPs at the concentration of $0.25 \%$, the liposomes transform into multilayered structures, as shown in Figure 4b, with a clearer visualization in the inset to Figure $4 \mathrm{~b}$. We again attribute this formation of multilayered structures to the breakage of some of the large unextruded vesicles into HMP supported fragments which
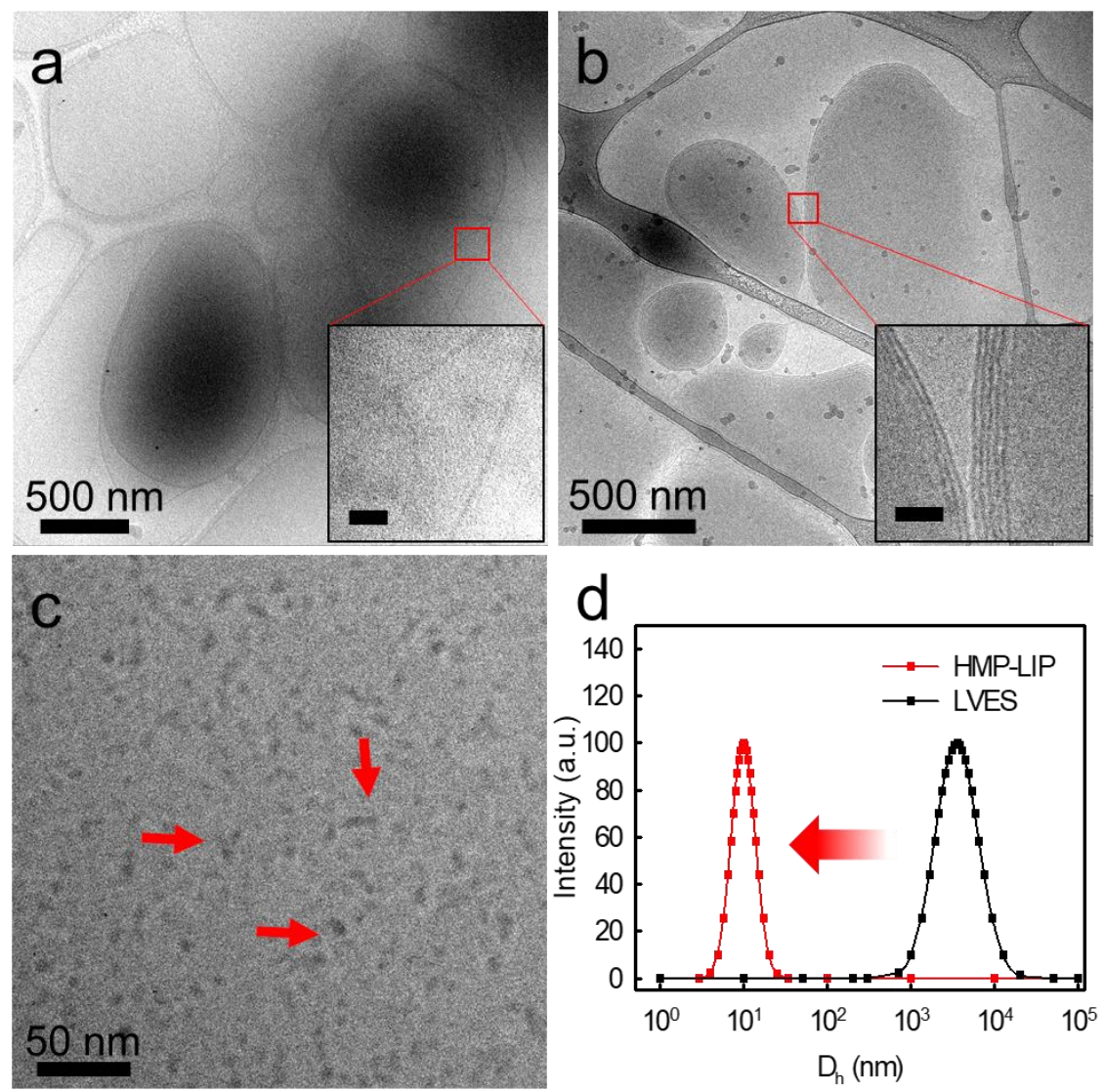

Figure 4. CryoTEM images of $0.25 \%$ large unextruded vesicles incubated with HMP at different concentrations and the size distributions of such large vesicles and HMP-lipid fragments. (a) Large vesicles with the size of several microns are formed from thin film hydration (LVES). (b) Large vesicles with multiple layers are formed with the addition of $0.25 \%$ HMP. The scale bars in the insets are $50 \mathrm{~nm}$. (c) Vesicles are broken into small fragments with the addition of $0.5 \%$ HMP. (d) The size distribution shows an effective vesicle diameter of $3.3 \mu \mathrm{m}$, which shifts to $9.9 \mathrm{~nm}$ with polydispersity index 0.13 upon the addition of $0.5 \%$ HMP leading to the formation of HMP-lipid fragments. 
anchor to intact vesicles through hydrophobe insertion. Further addition of the HMP to the concentration of $0.5 \%$ results in disruption of the entire population of large unextruded vesicles into small fragments as shown in Figure 4c. We have also carried out DLS measurement of the unextruded vesicular system before and after the addition of HMPs. The reduction in mean hydrodynamic diameter from $3.5 \mu \mathrm{m}$ prior to breakage to $9.9 \mathrm{~nm}$ with polydispersity index 0.13 after breakage again verifies this disruption of the liposomes into small HMP-lipid fragments.

The rupture of the large unextruded vesicles was also characterized by optical and fluorescence microscopy. The vesicles were prepared from PC lipid with 0.3 mol\% 1,2-dioleoyl-

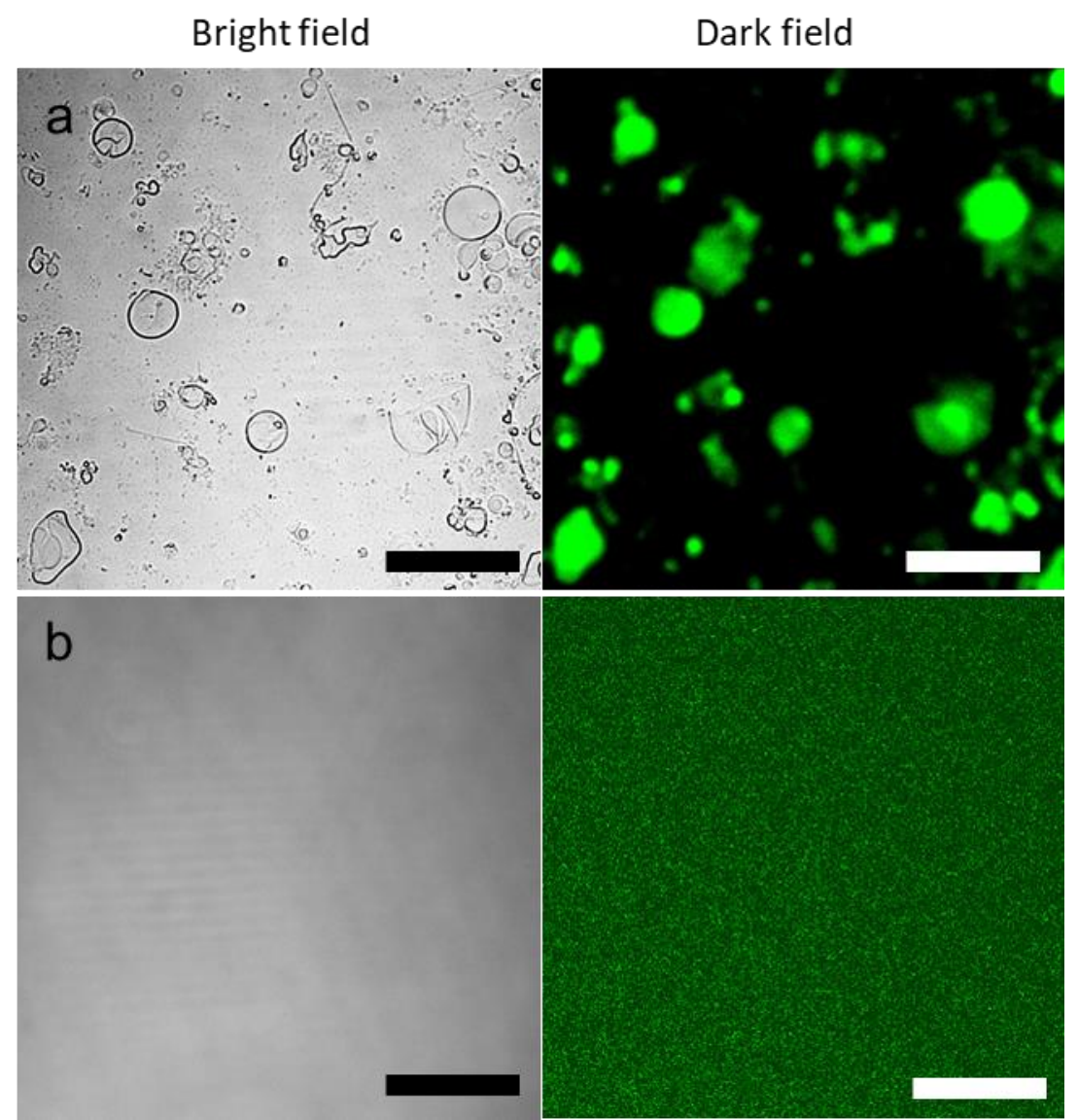

Figure 5. Bright field and fluorescence microscopy of fluorescently tagged $(0.3 \mathrm{~mol} \%)$ large unextruded vesicles and HMP-lipid fragments. (a) The top row shows the images of the large unextruded vesicles prior to the addition of HMP. (b) The bottom row shows the complete disappearance of vesicles and the fluorescence images show the presence of small lipid structures throughout the field of vision. The scale bar is $100 \mu \mathrm{m}$ in all images. 
sn-glycero-3-phosphoethanolamine- $N$-(carboxyfluorescein) (PECF) to provide the fluorescent tag to the vesicles. The top row in Figure 5 shows the bright field and fluorescent images of the vesicles which can be distinctly seen in optical microscopy. The vesicles have irregular shapes due to the low melting transition temperature of PC lipids $\left(-15^{\circ} \mathrm{C}\right)$ allowing shape flexibility at ambient temperature. All vesicles emit fluorescence when irradiated at $488 \mathrm{~nm}$ indicating that they are tagged by the PECF. The images in the bottom row show the complete disappearance of liposomes. The fluorescence signal is spread over the entire background indicating the presence of small fluorescent entities throughout the field of vision. These results provide further evidence of unextruded vesicle rupture through the addition of HMP and the formation of HMP-lipid fragments.

Vesicle rupture was also examined through ${ }^{31} \mathrm{P}$ and ${ }^{1} \mathrm{H}$ NMR spectroscopy. Figure $6 \mathrm{a}$ shows the ${ }^{31} \mathrm{P}$ NMR spectra of liposomes incubated with different concentrations of HMPs. Without the addition of HMPs, the peak for phosphorus-31 is significantly line-broadened, characteristic of phospholipids immobilized in the bilayer structure with slow rotational rates. ${ }^{31}$ In the presence of $0.125 \%$ HMPs, the emergence of an isotropic peak is seen. With an increased HMP concentration of $0.25 \%$, the isotropic peak becomes more pronounced. With $0.5 \%$ HMPs where all the liposomes are broken into HMP-lipid fragments, the isotropic peak reveals rotational mobility of the small HMP-lipid fragments. At $0.125 \%$ HMP concentration, the emerged small peak is due to the break-up of the lipid bilayer and the formation of the HMP-lipid fragments. Because of the re-assembly of the fragment/HMP-lipid fragments onto the intact liposomes (the formation of multilayered liposomes), the isotropic peak is weak, but nevertheless indicates that there is rotational mobility of the lipids in the layers attached to bilayers of surviving intact liposomes. The increase in sharpness with increasing HMP is symptomatic of generating additional 
fragments through disruption of more liposomes. At a HMP concentration of $0.5 \%$, the strong and sharp isotropic peak is a reflection of the rotational mobility of the fully disrupted liposomes. ${ }^{32}$

a

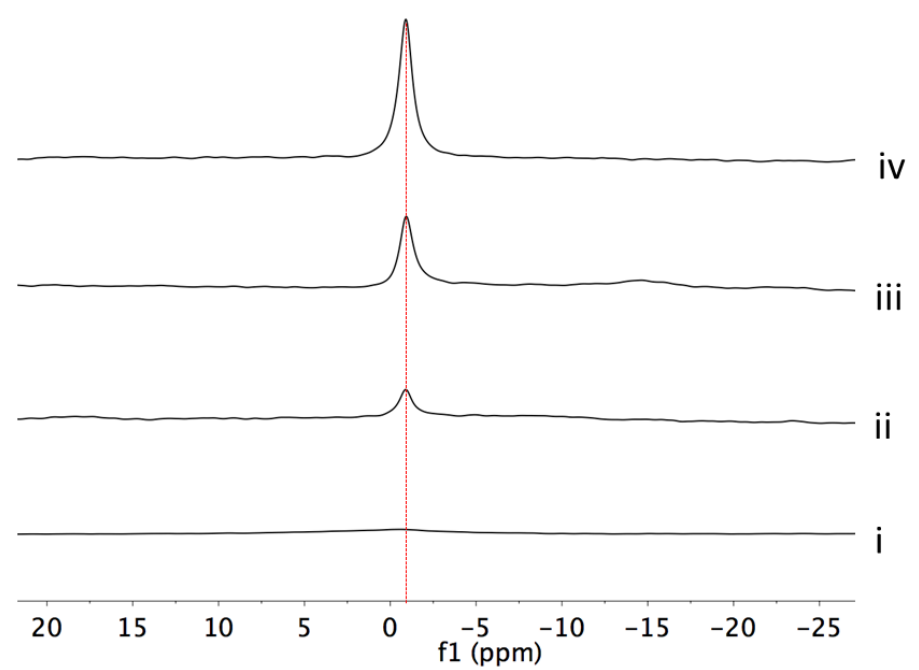

b

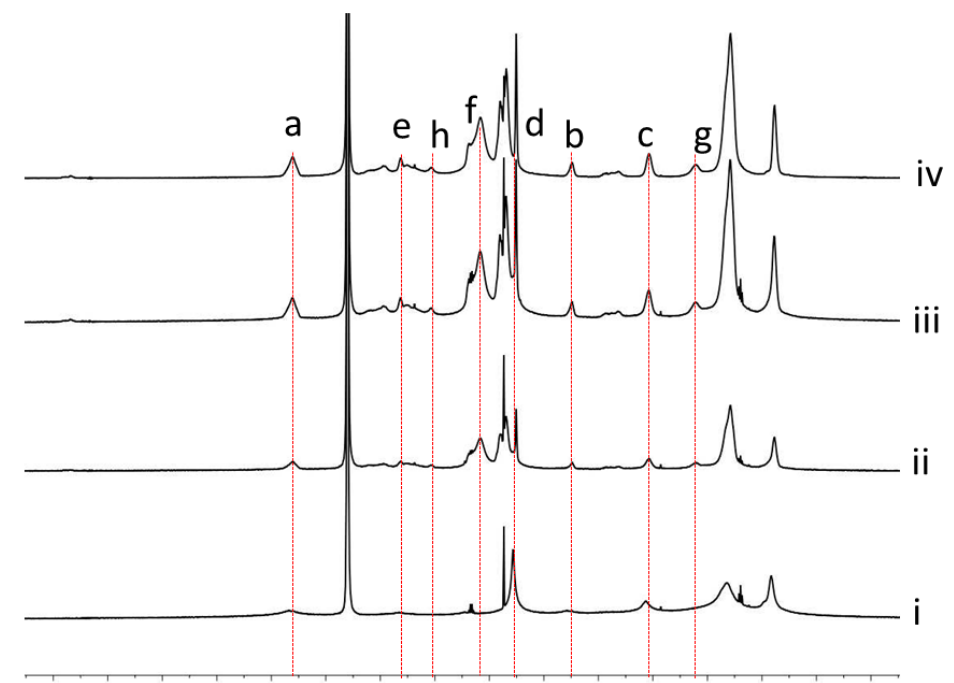

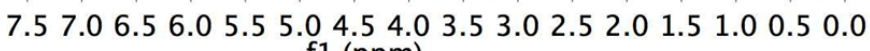

f1 (ppm)

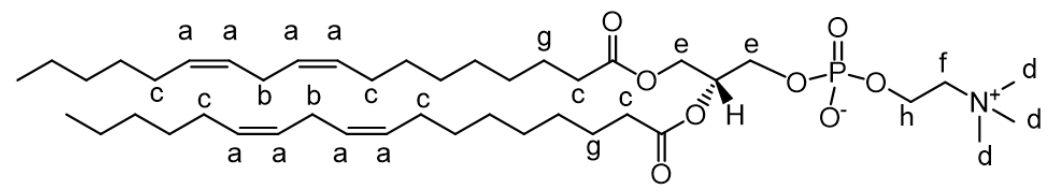

Figure 6. (a) ${ }^{31} \mathrm{P}$ NMR of vesicles with the addition of HMP at $0 \%$ (i), $0.125 \%$ (ii), $0.25 \%$ (iii), and $0.5 \%$ (iv). (b) ${ }^{1} \mathrm{H}$ NMR of vesicles with the addition of $\mathrm{HMP}$ at $0 \%$ (i), $0.125 \%$ (ii), $0.25 \%$ (iii), and $0.5 \%$ (iv). Peak sharpening is observed from both ${ }^{31} \mathrm{P}$ and ${ }^{1} \mathrm{H}$ NMR spectra with the increasing amount of HMP, indicating increased mobility of the lipid molecules. 
It is interesting to note that the normalized ratios of the integrated areas of the emergent ${ }^{31} \mathrm{P}$ resonances $(0.22 / 0.53 / 1)$ are very close to the HMP weight ratios used to rupture liposomes $(0.125 / 0.25 / 0.5$ or normalized to $0.25 / 0.5 / 1)$. If we assume that all liposomes are ruptured at the $0.5 \mathrm{wt} \%$ HMP level as indicated by the CryoTEM results, the normalized ratios of the integrated areas imply a retention of $78 \mathrm{~mol} \%$ of intact liposomes at an HMP concentration of $0.125 \mathrm{wt} \%$ and a retention of $47 \mathrm{~mol} \%$ of intact liposomes at an HMP concentration of $0.25 \mathrm{wt} \%$. ${ }^{1} \mathrm{H}$ NMR spectra in Figure $6 \mathrm{~b}$ also show the emergence of sharpened peaks of the protons in the alkyl chains of the lipid molecules. The protons at 5.4-5.7 $\mathrm{ppm}\left(-\mathrm{CH}_{2} \mathrm{CHCHCH}_{2}-\right.$ a), 2.7-2.8 ppm ($\mathrm{CHCH}_{2} \mathrm{CH}-$, b), and 1.9-2.2 ppm (- $\mathrm{CHCH}_{2} \mathrm{CH}_{2}-$ and $\mathrm{CH}_{2} \mathrm{CH}_{2} \mathrm{COO}-$, c) belong exclusively to the lipid and do not interfere with the protons from HMP (Supporting Information S4, Figure S3). ${ }^{33}$ The sharpening of these peaks upon the addition of HMPs implies the formation of small fragments of HMPs and phospholipids. The peak growth of other protons in the lipid molecules overlap with HMP protons and are not specifically analyzed for enhanced rotational mobility through vesicle rupture.

\section{Hydrophobic Drug Encapsulation in the HMP-lipid Fragment}

Sorafenib (SF) is a tyrosine protein kinase inhibitor and is currently FDA approved for the treatment of renal cell carcinoma, thyroid cancer, and liver cancer. Sorafenib inhibits protein kinases such as VEGFR, PDGFR, and the Raf family of kinases thus suppressing angiogenesis and inducing apoptosis. ${ }^{34}$ However, it is a highly hydrophobic compound with a very low solubility in water of $0.2 \mu \mathrm{g} \mathrm{mL} \mathrm{m}^{-1} .{ }^{35}$ This leads to large dose requirements with attendant physiological and psychosomatic side effects. ${ }^{36}$ To improve the bioefficiency of Sorafenib delivery, nanocarriers including liposomes have been used where Sorafenib has been solubilized in the hydrophobic lipid bilayer. ${ }^{27,37}$ 
In this work, we have used SF as a model component to demonstrate the ability of the HMP-lipid fragment to encapsulate such hydrophobic drugs. Figure $7 \mathrm{a}$ is a visual observation of various SF samples, (i) dispersed in water leading to rapid precipitation (SF) (ii) in large unextruded vesicles (LVES-SF) (iii) in the extruded liposomes (LIPO-SF) and (iv, v) in HMPlipid fragments (HMP-LIP-SF and HMP-LIP-SF-P), respectively. We note the turbidity of the large unextruded vesicle system (LVES-SF) and the translucency of the liposome suspension containing SF (LIPO-SF). HMP-LIP-SF and HMP-LIP-SF-P are distinct samples that need clarification. HMP-LIP-SF is obtained by adding 0.5 wt $\%$ HMP to a solution of large unextruded vesicles containing SF and rupturing these vesicles to form the fragments containing SF. HMPLIP-SF-P is an alternative method of forming fragments where the HMP is added directly to the lipid and SF in the chloroform-methanol solvent and plated out. Hydration is then carried out to form suspensions of the fragments. The initial amount of the SF added to the system were kept identical in all the samples. We note from visual observation that the HMP-LIP-SF-P solution is
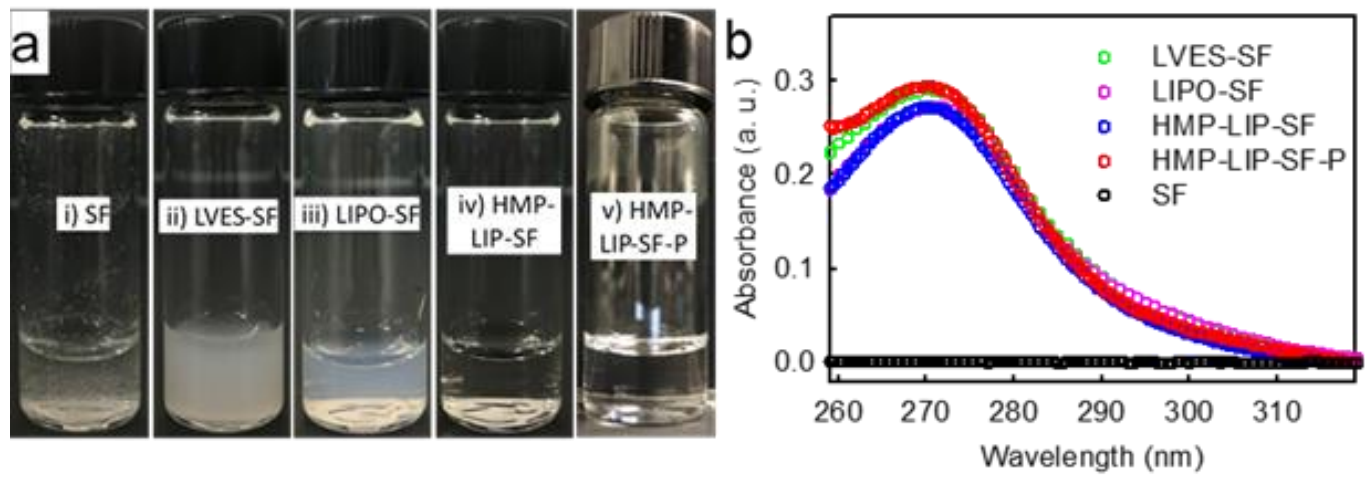

Figure 7. HMP-lipid fragments to sustain SF solubility in the aqueous phase. (a) Photographs of SF samples in water (SF), when sustained in large unextruded vesicle suspensions (LVES-SF), when encapsulated in extruded $100 \mathrm{~nm}$ liposomes (LIPO-SF), when encapsulated in HMP-lipid fragments by disrupting liposomes (HMP-LIP-SF), and when encapsulated directly in HMP-lipid fragments through the hydration of plated thin films containing HMP in addition to lipid and SF (HMP-LIP-SF-P). In all cases $0.5 \mathrm{wt} \% \mathrm{HMP}$ was used to form the fragments (b) UV spectrum of LVES-SF, LIPO-SF, HMP-LIP-SF, HMP-LIP-SF-P and supernatant of Sorafenib water suspension (SF). 
clear indicating that the direct incorporation of HMP into the plated thin film prevents the formation of vesicles upon hydration.

Figure $7 b$ illustrates the SF UV-Vis absorbance spectra for all samples described in Figure 7a, from which the concentrations of SF in these suspensions can be determined. The supernatant of each sample was diluted 20 times in DMSO to dissolve all components. Identical systems without SF were also diluted 20 times in DMSO and used as the spectrometer reference samples to subtract background absorbance. From Figure $7 \mathrm{~b}$, it is clear that the hydrophobic SF has insignificant solubility in aqueous solutions. However, all other lipid containing systems are able

Table I. SF Encapsulated in Large Vesicles, Liposomes, and HMP-lipid Fragments

\begin{tabular}{ccccc}
\hline & LVES-SF & LIPO-SF & HMP-LIP-SF $^{\mathrm{a}}$ & HMP-LIP-SF-P $^{\mathrm{b}}$ \\
\hline $\mathrm{C}_{\mathrm{SF}}\left(\mu \mathrm{g} \mathrm{mL}^{-1}\right)^{\mathrm{c}}$ & 59.9 & 56.8 & 56.6 & 59.7 \\
\hline $\mathrm{AE}(\%)^{\mathrm{d}}$ & 99.8 & 94.6 & 94.3 & 99.4 \\
\hline $\mathrm{DL}(\%)^{\mathrm{e}}$ & 2.40 & 2.27 & 2.26 & 2.39 \\
\hline
\end{tabular}

a, b: The concentration of HMP in the suspension is $0.5 \%$ by weight

c: Concentration of Sorafenib in the various suspensions. The initial concentration is 60 ( $\mu \mathrm{g}$ $\mathrm{mL}^{-1}$ )

d: Association efficiency (AE). $A E \%=W_{\text {Associated Drug }} / W_{\text {Total Drug }} \times 100 \%$, where $W_{\text {Associated Drug }}$ is the weight of drug that is associated with lipid bilayer, and $W_{\text {Total Drug }}$ is the weight of drug initially added to the flask.

e: Drug loading (DL). DL\% $=W_{\text {Associated Drug }} / W_{\text {Lipid }} \times 100 \%$, where $W_{\text {Lipid }}$ is the weight of total lipid.

to sustain SF in solution to approximately the same extent. Table I quantifies the concentration of

Sorafenib in these systems together with the encapsulation efficiencies and drug loading capacities.

It is observed that the HMP-lipid fragments prepared by rupturing large unextruded vesicles (HMP-LIP-SF) or by directly hydrating plated films containing lipids (HMP-LIP-SF-P) are able to stabilize SF in solution to the same level as liposomes (LIPO-SF) and large vesicles (LVES$\mathrm{SF})$. 
Figure 8 shows the CryoTEM images of the sample HMP-LIP-SF-P. The observation of small entities indicates that integration of HMP into the plated film prevents the folding up of the lipid layer into vesicles upon hydration. The DLS data of Figure $8 \mathrm{~b}$ indicates the size
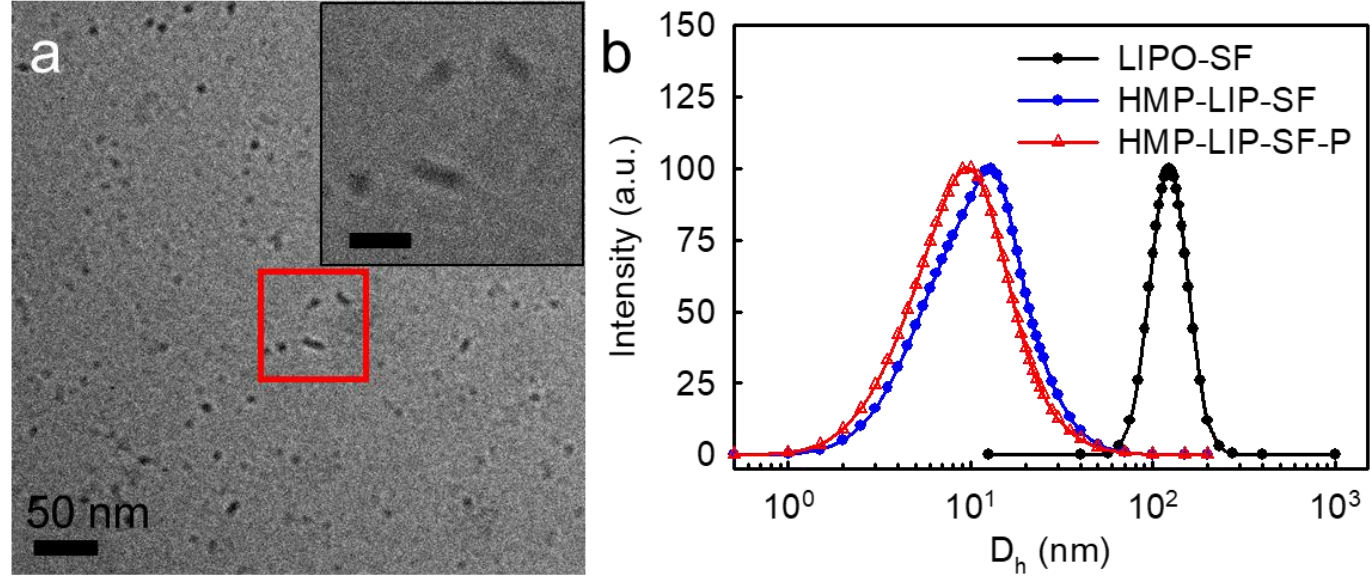

Figure 8. HMP-lipid fragments (HMP-LIP-SF-P) obtained from the thin film hydration method $(\mathrm{HMP} / \mathrm{lipid}=2 / 1, \mathrm{w} / \mathrm{w})$. The final HMP concentration in the solution is $0.5 \%$. (a) CryoTEM image of HMP-LIP-SF-P. The scale bar in the inset is $20 \mathrm{~nm}$. No lipid vesicles are observed, suggesting that HMP in the plated lipid thin film prevents the formation of lipid vesicles. (b) Size distribution of LIPO-SF, HMP-LIP-SF and HMP-LIP-SF-P from DLS showing similarity in the size range of HMP-lipid-sorafenib fragments prepared either through liposome disruption (HMP-LIP-SF) or through hydration of the plated components.

characteristics of fragments prepared by disrupting Sorafenib containing vesicles (HMP-LIP-SF) and prepared by hydrating plated out films containing lipid, HMP and Sorafenib (HMP-LIP-SFP). Both systems show similar size characteristics with mean of $11.2 \mathrm{~nm}$ and $9.1 \mathrm{~nm}$, and PDI values of 0.35 and 0.4 respectively. We note that there is a broader size distribution of the systems containing SF in comparison to the SF-free systems of Figures 3 and 4.

The prevention of vesicle formation and the direct formation of small polymer+lipid entities containing hydrophobic drugs is of direct interest in designing nanoscale carriers. We have done experiments with decreasing the HMP level to determine an approximate HMP concentration below which vesicle formation is unimpeded. The results are shown in Supporting Information S5 and we note that an HMP level of $0.25 \%$ (HMP/lipid ratio of 1/1) fully impedes vesicle formation 
while a lower HMP level of $0.125 \%$ allows some of the plated lipids to form vesicles upon hydration. As a conservative estimate, an HMP/lipid ratio of $1 / 1$ is necessary to entirely prevent vesicle formation upon hydration, and to generate HMP-lipid fragments containing SF.

\section{HMP-lipid Raft Attachment to Cells}

Our objective is to be able to use these HMP-lipid fragments containing drug species to be taken up into cells. In order to demonstrate the uptake of these fragments without disrupting cell function, we have done studies on cell membrane fusion and entry without the drug species, using a hepatocellular carcinoma cell line, Huh 7.5. ${ }^{38}$ A negative control sample was prepared by dissolving a fluorescent lipid 1, 2-dioleoyl-sn-glycero-3-phosphoethanolamine- $N$ carboxyfluorescein (PECF) in DMSO at $50 \mathrm{mM}$ which was then diluted with PBS. A Hoechst 33342 dye which binds only to the DNA was used for visualization of the nucleus. ${ }^{30}$ Figures $9 \mathrm{a}$ and $9 \mathrm{~b}$ show optical and fluorescence microscopy images respectively, of living cells incubated with the negative control sample. In Figure 9b, the blue fluorescence indicates the cells are viable when incubated with the negative control sample. Additionally, we notice the very weak green fluorescence observed around the nucleus revealing the location of PECF molecules that have entered the cell. Figure $9 \mathrm{c}$ and $9 \mathrm{~d}$ show the corresponding optical and fluorescence microscopy images of the cells incubated with the PECF-labeled HMP-lipid fragments prepared by hydrating thin films of HMP, PECF and PC to obtain fragments in solution containing $0.25 \mathrm{wt} \%$ of HMP and $0.25 \mathrm{wt} \%$ of lipids with the PECF component comprising $0.3 \mathrm{~mol} \%$ of the total lipid content (HMP-PECF+LIP-P). The final PECF concentration in HMP-PECF+LIP-P sample was kept the same as that of the negative control sample. Cells incubated with HMP-PECF+LIP-P show an intense green fluorescence in comparison with the negative control. The strong fluorescence indicates efficient attachment and possible uptake of the HMP-PECF+LIP-P fragment by Huh 7.5 
cells. The discrete fluorescent dots in the cell may possible indicate integration of the fluorescent lipid into the membranes of discrete organelles ${ }^{39}$ But we do note that based on the fluorescence images shown here, it is hard to determine whether the fragments are simply attached to the cell membrane or are actually endocytosed into the cell. Nevertheless, we can ascribe the efficient attachment of the fragments to hydrophobe insertion into the cell membrane. Initial cytotoxicity results (not shown) indicate that while these fragments are not intrinsically cytotoxic to the cell, the fragments with SF are extremely cytotoxic. Continuing work and a full study of the uptake of Sorafenib containing fragments by Huh 7.5 cells in inducing cell death is in progress. The major

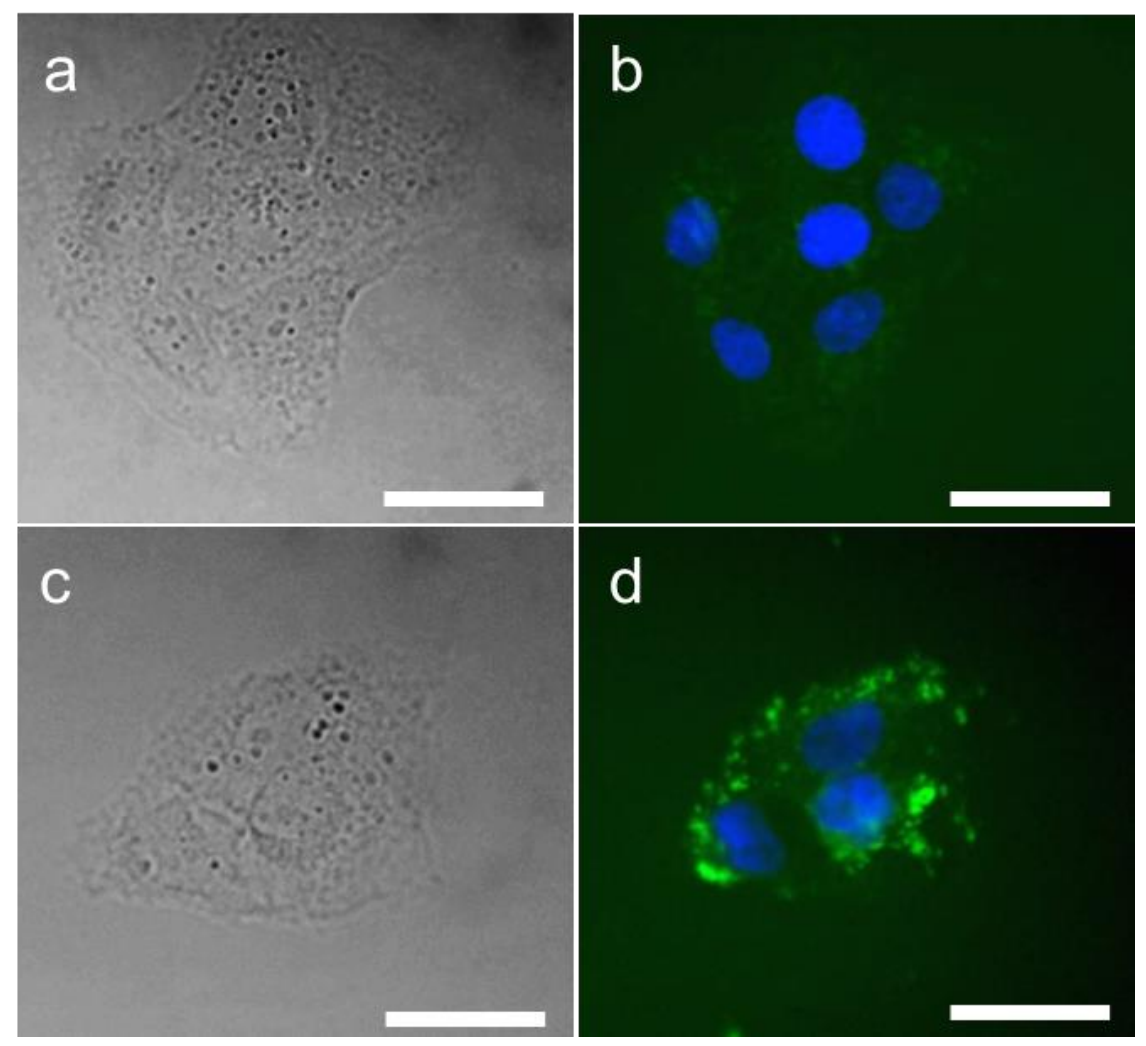

Figure 9. Optical and fluorescence microscopy of hepatocellular carcinoma cells (Huh 7.5 cell line). Cells were stained with Hoechst in PBS $\left(2 \mu \mathrm{g} \mathrm{mL}^{-1}\right)$ for nucleus visualization. Each scale bar presents $20 \mu \mathrm{m}$. (a and b) Hepatocellular carcinoma cells incubated with 1,2-dioleoyl-snglycero-3-phosphoethanolamine- $N$-carboxyfluorescein (PECF) alone. The sample was prepared by dissolving PECF in DMSO at $50 \mathrm{mM}$ followed by dilution with DI water. (c and d) Hepatocellular carcinoma cells incubated with HMP-PECF-LIP-P. The sample was prepared by hydrating the thin film containing $2.50 \mathrm{mg}$ HMP, $2.49 \mathrm{mg}$ soy PC lipid, and $0.01 \mathrm{mg}$ PECF using $1 \mathrm{~mL}$ DI water. 
aspects of the current study are simply to show that these HMP-lipid fragments generated through hydration of HMP-lipid plated out layers or through rupture of vesicles, are capable of encapsulating hydrophobic drug components and capable of attachment and entry to cells. 


\section{CONCLUSIONS}

This study indicates that the $N$-decyl hydrophobic side chains in the HMP backbone induce structural transformations of vesicles, regardless of vesicle size. The disruption of vesicle bilayers is a consequence of hydrophobe insertion into the bilayer at concentrations sufficient to create local instabilities of the bilayer and thus rupture the bilayer. While similar dissolutions of lipid bilayers can be accomplished with small molecule surfactants, ${ }^{40-41}$ we note the significant distinction here that entire fragments of bilayers can be broken off with such amphiphilic polymers in contrast to the use of detergents where vesicles transform to mixed micelles of lipids and detergents beyond a critical concentration of the detergent. ${ }^{42}$ The novelty of our observation is that at low concentration of HMP $(\leq 0.5 \%)$, the structures of either the liposome or the large vesicles undergo changes from unilamellar structures to multilamellar structures, while at high concentration of HMP $(>0.5 \%)$, the lipid bilayers form HMP-lipid fragments. These materials add to the class of small self-assembled structures that include mixed micelles and bicelles.

Such HMP-lipid fragments are promising candidates as nano-carriers for the delivery of hydrophobic drugs. Our results indicate that highly hydrophobic drugs such as Sorafenib can be directly incorporated into HMP-lipid fragments through integration of all components within a thin film plating which is then hydrated to form the fragments, leading to a one-step, quantitative encapsulation of Sorafenib in the HMP-lipid fragments. Uptake into cells appears to be fully feasible and we note that such uptake may be aided by dynamically free hydrophobes on HMP which may insert into cell membranes facilitating endocytic pathways for entry. Continuing studies will seek to establish the fundamentals of such uptake and to exploit the hydrophobic effect inherent in these nanoscale HMP-lipid fragments to enhance drug delivery. 
Supporting Information. S1. Synthetic routes of $N$-decyl NCA monomers, $N$-methoxyethyl NCA monomers, and hydrophobically modified polypeptoids (HMP); S2: ${ }^{1} \mathrm{H}$ NMR spectrum of HMP in $\mathrm{CD}_{2} \mathrm{Cl}_{2} ; \mathrm{S} 3$ :SEC chromatograms of HMP; $\mathrm{S} 4:{ }^{1} \mathrm{H}$ NMR spectra of liposome, HMP, and HMPliposome mixture in $\mathrm{D}_{2} \mathrm{O}$; S5:CryoTEM images of HMP-lipid fragments and vesicles formed through plated thin film of HMP and lipids. S6: Liposomes prepared through the freeze-thaw procedure as disrupted by HMP.

\section{ACKNOWLEDGEMENTS}

This work was supported by the U.S. Department of Energy under EPSCoR Grant No. DESC0012432. Additional funding was provided by the National Science Foundation Grant 1805608. 


\section{REFERENCES}

1. Allen, T. M.; Cullis, P. R., Liposomal Drug Delivery Systems: From Concept to Clinical Applications. Adv. Drug Deliv. Rev. 2013, 65 (1), 36-48.

2. Immordino, M. L.; Dosio, F.; Cattel, L., Stealth Liposomes: Review of the Basic Science, Rationale, and Clinical Applications, Existing and Potential. Int. J. Nanomedicine 2006, I (3), 297315 .

3. Torchilin, V. P., Recent Advances with Liposomes as Pharmaceutical Carriers. Nat. Rev. Drug Discov. 2005, 4 (2), 145-160.

4. $\quad$ Park, J. H.; Kim, H. A.; Cho, S. H.; Lee, M., Characterization of Hydrophobic Anti-cancer Drug-loaded Amphiphilic Peptides as a Gene Carrier. J. Cell. Biochem. 2012, 113 (5), 1645-1653. 5. Wu, M.; Ye, Z.; Liu, Y.; Liu, B.; Zhao, X., Release of Hydrophobic Anticancer Drug from a Newly Designed Self-assembling Peptide. Mol. BioSyst. 2011, 7 (6), 2040-2047.

6. $\quad$ Lin, L.; Wang, X.; Guo, Y.; Ren, K.; Li, X.; Jing, L.; Yue, X.; Zhang, Q.; Dai, Z., Hybrid Bicelles as a pH-sensitive Nanocarrier for Hydrophobic Drug Delivery. RSC Adv. 2016, 6 (83), 79811-79821.

7. $\quad$ Puri, A.; Loomis, K.; Smith, B.; Lee, J.-H.; Yavlovich, A.; Heldman, E.; Blumenthal, R., Lipid-Based Nanoparticles as Pharmaceutical Drug Carriers: From Concepts to Clinic. Crit. Rev. Ther. Drug Carrier Syst. 2009, 26 (6), 523-580.

8. Namiki, Y.; Fuchigami, T.; Tada, N.; Kawamura, R.; Matsunuma, S.; Kitamoto, Y.; Nakagawa, M., Nanomedicine for Cancer: Lipid-Based Nanostructures for Drug Delivery and Monitoring. Acc. Chem. Res. 2011, 44 (10), 1080-1093.

9. Danhier, F.; Feron, O.; Préat, V., To Exploit the Tumor Microenvironment: Passive and Active Tumor Targeting of Nanocarriers for Anti-cancer Drug Delivery. J. Control. Release 2010, 148 (2), 135-146.

10. Jain, A.; Agarwal, A.; Majumder, S.; Lariya, N.; Khaya, A.; Agrawal, H.; Majumdar, S.; Agrawal, G. P., Mannosylated Solid Lipid Nanoparticles as Vectors for Site-specific Delivery of an anti-cancer Drug. J. Control. Release 2010, 148 (3), 359-367.

11. Lahasky, S. H.; Hu, X.; Zhang, D., Thermoresponsive Poly(alpha-peptoid)s: Tuning the Cloud Point Temperatures by Composition and Architecture. ACS Macro Lett. 2012, 1 (5), 580584.

12. Gangloff, N.; Ulbricht, J.; Lorson, T.; Schlaad, H.; Luxenhofer, R., Peptoids and Polypeptoids at the Frontier of Supra- and Macromolecular Engineering. Chem. Rev. 2016, 116 (4), 1753-1802.

13. Sun, J.; Zuckermann, R. N., Peptoid Polymers: A Highly Designable Bioinspired Material. ACS Nano 2013, 7 (6), 4715-4732.

14. Zhang, D.; Lahasky, S. H.; Guo, L.; Lee, C.-U.; Lavan, M., Polypeptoid Materials: Current Status and Future Perspectives. Macromolecules 2012, 45 (15), 5833-5841.

15. Murnen, H. K.; Rosales, A. M.; Dobrynin, A. V.; Zuckermann, R. N.; Segalman, R. A., Persistence Length of Polyelectrolytes with Precisely Located Charges. Soft Matter 2013, 9 (1), 90-98.

16. Rosales, A. M.; Murnen, H. K.; Kline, S. R.; Zuckermann, R. N.; Segalman, R. A., Determination of the Persistence Length of Helical and Non-helical Polypeptoids in Solution. Soft Matter 2012, 8 (Copyright (C) 2016 American Chemical Society (ACS). All Rights Reserved.), 3673-3680. 
17. Miller, S. M.; Simon, R. J.; Ng, S.; Zuckermann, R. N.; Kerr, J. M.; Moos, W. H., Proteolytic Studies of Homologous Peptide and N-Substituted Glycine Peptoid Oligomers. Bioorganic Med. Chem. Lett. 1994, 4 (22), 2657-2662.

18. Xuan, S.; Lee, C.-U.; Chen, C.; Doyle, A. B.; Zhang, Y.; Guo, L.; John, V. T.; Hayes, D.; Zhang, D., Thermoreversible and Injectable ABC Polypeptoid Hydrogels: Controlling the Hydrogel Properties through Molecular Design. Chem. Mater. 2016, 28 (3), 727-737.

19. Lu, L.; Lahasky, S. H.; Zhang, D.; Garno, J. C., Directed Growth of Polymer Nanorods Using Surface-Initiated Ring-Opening Polymerization of N-Allyl N-Carboxyanhydride. ACS Appl. Mater. Interfaces 2016, 8 (6), 4014-4022.

20. Hörtz, C.; Birke, A.; Kaps, L.; Decker, S.; Wächtersbach, E.; Fischer, K.; Schuppan, D.; Barz, M.; Schmidt, M., Cylindrical Brush Polymers with Polysarcosine Side Chains: A Novel Biocompatible Carrier for Biomedical Applications. Macromolecules 2015, 48 (7), 2074-2086.

21. Chen, Y.; Javvaji, V.; MacIntire, I. C.; Raghavan, S. R., Gelation of Vesicles and Nanoparticles using Water-Soluble Hydrophobically Modified Chitosan. Langmuir 2013, 29 (49), 15302-15308.

22. Lee, J.-H.; Gustin, J. P.; Chen, T.; Payne, G. F.; Raghavan, S. R., Vesicle-Biopolymer Gels: Networks of Surfactant Vesicles Connected by Associating Biopolymers. Langmuir 2005, 21 (1), 26-33.

23. Zhang, Y.; Xuan, S.; Owoseni, O.; Omarova, M.; Li, X.; Saito, M. E.; He, J.; McPherson, G. L.; Raghavan, S. R.; Zhang, D.; John, V. T., Amphiphilic Polypeptoids Serve as the Connective Glue to Transform Liposomes into Multilamellar Structures with Closely Spaced Bilayers. Langmuir 2017, 33 (11), 2780-2789.

24. Lee, C.-U.; Smart, T. P.; Guo, L.; Epps, T. H.; Zhang, D., Synthesis and Characterization of Amphiphilic Cyclic Diblock Copolypeptoids from N-Heterocyclic Carbene-Mediated Zwitterionic Polymerization of N-Substituted N-carboxyanhydride. Macromolecules 2011, 44 (24), 9574-9585.

25. Xuan, S.; Gupta, S.; Li, X.; Bleuel, M.; Schneider, G. J.; Zhang, D., Synthesis and Characterization of Well-Defined PEGylated Polypeptoids as Protein-Resistant Polymers. Biomacromolecules 2017, 18 (3), 951-964.

26. Holder, G. E.; McGary, C. M.; Johnson, E. M.; Zheng, R.; John, V. T.; Sugimoto, C.; Kuroda, M. J.; Kim, W.-K., Expression of the Mannose Receptor CD206 in HIV and SIV Encephalitis: A Phenotypic Switch of Brain Perivascular Macrophages with Virus Infection. J. Neuroimmune Pharmacol. 2014, 9 (5), 716-726.

27. Arora, J. S.; Murad, H. Y.; Ashe, S.; Halliburton, G.; Yu, H.; He, J.; John, V. T.; Khismatullin, D. B., Ablative Focused Ultrasound Synergistically Enhances Thermally Triggered Chemotherapy for Prostate Cancer in Vitro. Mol. Pharm. 2016, 13 (9), 3080-3090.

28. Castile, J. D.; Taylor, K. M. G., Factors affecting the size distribution of liposomes produced by freeze-thaw extrusion. Int J Pharm 1999, 188 (1), 87-95.

29. Costa, A. P.; Xu, X. M.; Burgess, D. J., Freeze-Anneal-Thaw Cycling of Unilamellar Liposomes: Effect on Encapsulation Efficiency. Pharm Res-Dordr 2014, 31 (1), 97-103.

30. Chazotte, B., Labeling Nuclear DNA with Hoechst 33342. Cold Spring Harb. Protoc. 2011, 83-85.

31. Oluwole, A. O.; Danielczak, B.; Meister, A.; Babalola, J. O.; Vargas, C.; Keller, S., Solubilization of Membrane Proteins into Functional Lipid-Bilayer Nanodiscs Using a Diisobutylene/Maleic Acid Copolymer. Angew. Chem. Int. Ed. 2017, 56 (7), 1919-1924. 
32. Traïkia, M.; Warschawski, D. E.; Recouvreur, M.; Cartaud, J.; Devaux, P. F., Formation of Unilamellar Vesicles by Repetitive Freeze-Thaw Cycles: Characterization by Electron Microscopy and 31p-Nuclear Magnetic Resonance. Eur. Biophys. J. 2000, 29 (3), 184-195.

33. Hong, M.; Schmidt-Rohr, K.; Nanz, D., Study of phospholipid structure by 1H, 13C, and 31P dipolar couplings from two-dimensional NMR. Biophys. J. 1995, 69 (5), 1939-1950.

34. Liu, L.; Cao, Y.; Chen, C.; Zhang, X.; McNabola, A.; Wilkie, D.; Wilhelm, S.; Lynch, M.; Carter, C., Sorafenib Blocks the RAF/MEK/ERK Pathway, Inhibits Tumor Angiogenesis, and Induces Tumor Cell Apoptosis in Hepatocellular Carcinoma Model PLC/PRF/5. Cancer Res. 2006, 66 (24), 11851.

35. Wang, X.-q.; Fan, J.-m.; Liu, Y.-o.; Zhao, B.; Jia, Z.-r.; Zhang, Q., Bioavailability and Pharmacokinetics of Sorafenib Suspension, Nanoparticles and Nanomatrix for Oral Administration to Rat. Int. J. Pharm. 2011, 419 (1), 339-346.

36. Kumar, R. J.; Barqawi, A.; Crawford, E. D., Adverse Events Associated with Hormonal Therapy for Prostate Cancer. Nat. Rev. Urol 2005, 7 (Suppl 5), S37-S43.

37. Liu, J.; Boonkaew, B.; Arora, J.; Mandava, S. H.; Maddox, M. M.; Chava, S.; Callaghan, C.; He, J.; Dash, S.; John, V. T.; Lee, B. R., Comparison of Sorafenib-Loaded Poly (Lactic/Glycolic) Acid and DPPC Liposome Nanoparticles in the in Vitro Treatment of Renal Cell Carcinoma. J. Pharm. Sci. 2015, 104 (3), 1187-1196.

38. Chandra, P. K.; Bao, L.; Song, K.; Aboulnasr, F. M.; Baker, D. P.; Shores, N.; Wimley, W. C.; Liu, S.; Hagedorn, C. H.; Fuchs, S. Y.; Wu, T.; Balart, L. A.; Dash, S., HCV Infection Selectively Impairs Type I but Not Type III IFN Signaling. Am. J. Pathol. 2014, 184 (1), 214-229. 39. Zhu, L.; Simpson, J. M.; Xu, X.; He, H.; Zhang, D.; Yin, L., Cationic Polypeptoids with Optimized Molecular Characteristics toward Efficient Nonviral Gene Delivery. ACS Appl. Mater. Interfaces 2017, 9 (28), 23476-23486.

40. Lichtenberg, D.; Ahyayauch, H.; Goni, F. M., The Mechanism of Detergent Solubilization of Lipid Bilayers. Biophys. J. 2013, 105 (2), 289-299.

41. Lichtenberg, D.; Ahyayauch, H.; Alonso, A.; Goñi, F. M., Detergent Solubilization of Lipid bilayers: a Balance of Driving Forces. Trends Biochem. Sci. 2013, 38 (2), 85-93.

42. Almgren, M., Mixed Micelles and Other Structures in the Solubilization of Bilayer Lipid Membranes by Surfactants. Biochim. Biophys. Acta. 2000, 1508 (1-2), 146-163. 